\title{
The Apparent Thermal Radiation Properties of an Isothermal V-Groove With Specularly Reflecting Walls
}

\author{
Richard B. Zipin*
}

(July 26, 1966)

\begin{abstract}
The reflection of a parallel beam of light incident in a $V$-groove is treated for the case when the walls are specularly reflecting. Methods of calculating apparent reflectances and absorptances are given. Experiments have been performed to test the theoretical calculations, and the agreement has been found to be satisfactory.
\end{abstract}

Key Words: Reflectance, specular walls, thermal radiation, V-grooves.

\section{Introduction}

Recently there has been a great deal of work reported in the literature on the problem of predicting the apparent thermal radiation properties of cavities and rough surfaces when the properties of ideally smooth surfaces of the same material are known. An interesting problem that has not been previously treated very completely is reflection by an isothermal $\checkmark$-groove, whose walls are specularly reflecting. Sparrow and Lin [3] ${ }^{1}$ have reported some results for the effective absorptance of such a groove. Their method of analysis is followed here using a simpler procedure, extended somewhat, and results are given for effective directional reflectance.

The main limitation on the results reported here is due to the assumption that the $V$-groove has specular walls. This assumption is valid when the wavelength of the incident radiation is much less than the characteristic dimensions of the groove, and at the same time much greater than the characteristic dimensions of the roughness of the groove walls. Experimental results reported by the author [4] have shown this to be true.

\section{Reflection Within a V-Groove With Specularly Reflecting Walls}

The problem to be considered is illustrated in figure 1. The $\vee$-groove has an opening angle of $2 \theta$, a depth $h$, and is infinite in the direction normal to the plane of incidence. The method of calculation is based on the fact that light reflected from a specular surface appears to come from an image located

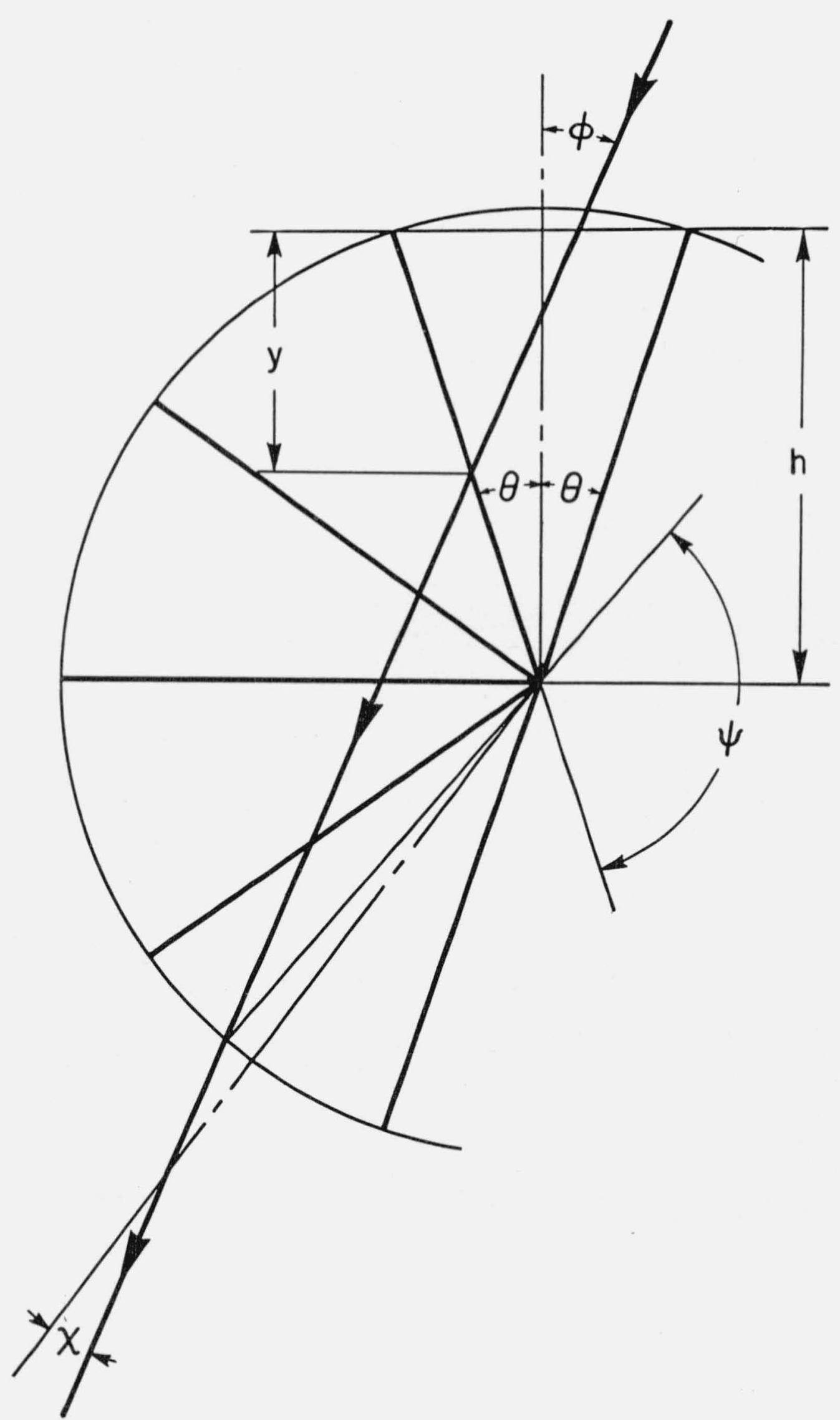

FIGURE 1. A single incident beam being specularly reflected within a V-groove.

\footnotetext{
*Present address: The Sheffield Corporation, Dayton, Ohio.

${ }^{1}$ Figures in brackets indicate the literature references at the end of this paper
} 
behind the surface at a distance equal to the distance of the source from the front of the specular surface [2]. The number of reflections, $n$, that an incident beam makes at the walls of the groove before it leaves determines the apparent thermal radiation properties exhibited by the groove, and this may be determined by counting the number of mirror images of the first surface that are crossed by an undeviated beam. A single beam is incident in the groove at an acute angle $\phi$ measured with respect to the normal to the groove opening, and leaves the groove at an acute angle $\chi$ measured with respect to the same normal.

The number of reflections is given by

$$
n=\left[\frac{\psi}{2 \theta}\right]
$$

where $\psi$ is defined in figure 1 , and the square brackets indicate that the quantity enclosed within them should be rounded off to the nearest higher integer. This is because there can never be a fraction of a reflection, and all beams incident in a groove must make at least one reflection.

There are two separate cases to be distinguished here:

(1) If $\phi>\theta$, an incident beam can only strike the far wall on entering the groove, and,

(2) if $\phi<\theta$, an incident beam may strike either wall on entering the groove.

For beams incident on the far wall, the law of sines gives

$$
\frac{h / \cos \theta}{\sin (\theta+\phi)}=\frac{\left(h-y_{f}\right) / \cos \theta}{\sin \left(\pi-\psi_{f}-\theta-\phi\right)}
$$

where $y$ is the vertical distance from the groove opening at which a beam strikes the wall, and the subscript $f$ indicates a quantity associated with a beam incident on the far wall. Now,

$$
\psi_{f}=\pi-\theta-\phi-\sin ^{-1}\left\{\left(1-\frac{y_{f}}{h}\right) \sin (\theta+\phi)\right\}
$$

and by geometry

$$
\chi_{f}=(-1)^{n}\left(\pi-\phi-2 n_{f} \theta\right) .
$$

For beams incident on the near wall, the subscript $n$ is used, and

$$
\frac{h / \cos \theta}{\sin (\theta-\phi)}=\frac{\left(h-y_{n}\right) / \cos \theta}{\sin \left(-\pi+\psi_{n}+\theta+\phi\right)}
$$

so that

$$
\psi_{n}=\pi-\theta-\phi+\sin ^{-1}\left\{\left(1-\frac{y_{n}}{h}\right) \sin (\theta-\phi)\right\}
$$

and in this case

$$
\chi_{n}=(-1)^{n-1}\left(\pi+\phi-2 n_{n} \theta\right) .
$$

A value of $\chi$ obtained from either of eqs (4) and (7) which is equal to or greater than $90^{\circ}$ is obviously forbidden unless $\phi=90^{\circ}$.

By the use of eq (1), with eqs (3) and (4) or eqs (6) and (7), the reflections undergone by any beam incident in the groove may be determined.

If the groove is fully illuminated and $\phi<\theta$ both walls will have energy directly incident on them, but if $\phi>\theta$ only the far wall will receive energy by direct incidence. In the latter case the far wall will be directly illuminated from $y=0$ to $y=y_{\max }$, where $y_{\max }$ is the value of $y$ at which the incident beam that just grazes the lip of the groove on entering strikes the far wall. To calculate $y_{\max }$, the law of sines is used again, and

$$
\frac{h / \cos \theta}{\sin (\pi-\theta-\phi)}=\frac{\left(h-y_{\max }\right) / \cos \theta}{\sin (\phi-\theta)} .
$$

Therefore,

$$
y_{\text {max }}=\frac{2 h \cos \phi \sin \theta}{\sin (\theta+\phi)}
$$

and when $\phi=\theta, y_{\max }=h$ as required.

All beams incident on both the near and the far walls of the groove will undergo the same number of reflections and leave the groove at the same angle as either the $y=0$ or the $y=y_{\max }$ beams. $^{2} \quad$ The subscripts 0 and max denote quantities related to the beams incident at $y=0$ and $y=y_{\max }$ respectively, and $y_{c}$ will denote the critical value of $y$ at which $n$ changes from $n_{0}$ to $n_{\max }$ and $\chi$ changes from $\chi_{0}$ to $\chi_{\max }$. A beam incident at $y_{c}$ will just graze the lip of the groove on leaving. For the far wall, the law of sines is again used, and

$$
\frac{h / \cos \theta}{\sin (\theta+\phi)}=\frac{\left(h-y_{c, f}\right) / \cos \theta}{\sin \left(\pi-2 n_{0, f} \theta-\theta-\phi\right)}
$$

so that

$$
y_{c, f}=h\left\{1-\frac{\sin \left(2 n_{0, f} \theta+\theta+\phi\right)}{\sin (\theta+\phi)}\right\} .
$$

${ }^{2}$ For the near wall, $y_{\max }$ must be $h$. 
For the near wall,

$$
\frac{h / \cos \theta}{\sin (\theta-\phi)}=\frac{\left(h-y_{c, n}\right) / \cos \theta}{\sin \left(\pi-2 n_{0, n} \theta-\theta+\phi\right)}
$$

and so

$$
\dot{y}_{c, n}=h\left\{1-\frac{\sin \left(2 n_{0, n} \theta+\theta-\phi\right)}{\sin (\theta-\phi)}\right\}
$$

If $\phi=0$, there is no distinction between the near and far walls, and eqs (11) and (13) give identical results.

The angle $\chi$ will be said to be negative if it is measured in the same direction from the normal as $\phi$ is, and positive if it is measured in the opposite direction. When $\phi>\theta, \chi$ is positive if $n$ is even and negative when $n$, is odd.

\section{The Calculation of the Apparent Thermal Radiation Properties}

If the average reflectance of the groove walls is $\rho$, and the walls are opaque, then the average absorptance of the walls is $\alpha=1-\rho$. When a single narrow beam of energy per unit time $I_{i}$ is incident in the groove at an angle $\phi>\theta$, it will strike the far wall, be reflected $n$ times within the groove, and then leave at an angle $\chi$. $\chi$ will have one of the two values $\chi_{0}$ or $\chi_{\max }$, according to the value of $y$ at which the beam is incident.

On the first reflection of the beam, $(1-\rho) I_{i}$ is absorbed and $\rho I_{i}$ is reflected. On the second reflection, $(1-\rho) \rho I_{i}$ is absorbed and $\rho^{2} I_{i}$ is reflected. This continues until the final, $n$th reflection, when $(1-\rho) \rho^{n-1} I_{i}$ is absorbed and $\rho^{n} I_{i}$ is reflected. The energy-rate of the beam leaving the groove after the $n$ reflections is therefore $I_{r}=\rho^{n} I_{i}$.

If the effective reflectance is defined as the ratio of the energy reflected at the angle $\chi$ to the energy incident at the angle $\phi$, it is given by

$$
\rho_{\mathrm{eff}}(\phi, \chi)=\frac{I_{r}}{I_{i}}=\rho^{n}
$$

where the value of $n$ used must correspond to the value $x$ considered.

If $\phi<\theta$, a single narrow beam may strike either the near or the far wall of the groove. In this case $\chi$ will have one of the four values, $\chi_{0, f}, \chi_{\max , f}, \chi_{0, n}$, or $\chi_{\max , n}$ Equation (14) may be used to find the effective reflectance.

Now consider the groove to be fully illuminated. If $\phi>\theta$, energy is incident on the far wall from $y=0$ to $y=y_{\max }$. Let the incident beam carry energy per unit time $I_{i}$ per unit width in the plane of incidence and normal to its direction of propagation, or equivalently $I_{i} \sin (\theta+\phi)$ per unit width along the groove wall, so that the total energy incident in the groove per unit time is

$$
I(\phi>\theta)=I_{i} \sin (\theta+\phi) y_{\max } / \cos \theta .
$$

Recalling eq (9), and substituting for $y_{\max }$, this becomes

$$
I(\phi>\theta)=2 I_{i} h \cos \phi \tan \theta .
$$

The fraction of this energy that leaves after $n_{0}$ reflections at an angle $\chi_{0}$ is $\frac{y_{c}}{y_{\max }} \rho^{n_{0}}$, while the fraction that leaves after $n_{\max }$ reflections at $\chi_{\max }$ is $\left(1-\frac{y_{c}}{y_{\max }}\right) \rho^{n} \max$.

As before, the effective reflectance at an angle $\chi$ will be defined as the ratio of the energy leaving the groove in the direction $\chi$ to the total energy incident in the groove. In this case we have two such reflectances,

$$
\rho_{\text {eff }}\left(\phi, \chi_{0}\right)=\frac{y_{c}}{y_{\max }} \rho^{n_{0}}
$$

and

$$
\rho_{\text {eff }}\left(\phi, \chi_{\max }\right)=\left(1-\frac{y_{c}}{y_{\max }}\right) \rho^{n} \max
$$

Since the total energy reflected by the groove is the sum of these two reflectances multiplied by the incident energy, the total energy absorbed is

$$
\left\{1-\rho_{\text {eff }}\left(\phi, \chi_{0}\right)-\rho_{\text {eff }}\left(\phi, \chi_{\max }\right)\right\} I(\phi>\theta)
$$

and so the effective absorptance is

$$
\alpha_{\text {eff }}(\phi>\theta)=1-\rho_{\text {eff }}\left(\phi, \chi_{0}\right)-\rho_{\text {eff }}\left(\phi, \chi_{\max }\right) .
$$

This expression is only a function of $\phi$.

If $\phi<\theta$ and the groove is fully illuminated, energy is incident on both walls from $y=0$ to $y=h$. If the incident beam carries energy per unit time $I_{i}$ per unit width in the plane of incidence and normal to its direction of propagation, then it will have $I_{i} \sin (\theta+\phi)$ per unit width along the far wall and $I_{i} \sin (\theta-\phi)$ per unit width along the near wall. The total energy incident on the far wall is $I_{i} \sin (\theta+\phi) h / \cos \theta$ and on the near wall is $I_{i} \sin (\theta-\phi) h / \cos \theta$, so that the total energy incident in the groove is

$$
I(\phi<\theta)=2 I_{i} h \cos \phi \tan \theta=I(\phi>\theta) .
$$

From the above it is easily seen that the fraction of the total energy incident in the groove that is directly incident on the near wall is $\frac{\sin (\theta-\phi)}{2 \sin \theta \cos \phi}$ and the fraction of this energy that leaves after $n_{0, n}$ reflections at $\chi_{0, n}$ is $\frac{y_{c, n}}{h} \rho^{n} 0, n$ and that leaves after $n_{\max , n}$ reflec- 
tions at $\chi_{\max , n}$ is $\left(1-\frac{y_{c, n}}{h}\right) \rho^{n} \max , n$. The fraction of the total energy incident in the groove that is directly incident on the far wall is $\frac{\sin (\theta+\phi)}{2 \sin \theta \cos \phi}$. The effective reflectances are, therefore, given by

$$
\begin{aligned}
\rho_{\mathrm{eff}}\left(\phi, \chi_{0, n}\right) & =\frac{\sin (\theta-\phi)}{2 \sin \theta \cos \phi} \frac{y_{c, n}}{h} \rho^{n_{0, n}} \\
\rho_{\mathrm{eff}}\left(\phi, \chi_{\max , n}\right) & =\frac{\sin (\theta-\phi)}{2 \sin \theta \cos \phi}\left(1-\frac{y_{c, n}}{h}\right) \rho^{n_{\max , n}} \\
\rho_{\mathrm{eff}}\left(\phi, \chi_{0, f}\right) & =\frac{\sin (\theta+\phi)}{2 \sin \theta \cos \phi} \frac{y_{c, f}}{h} \rho^{n_{0, f}}
\end{aligned}
$$

and

$\rho_{\mathrm{eff}}\left(\phi, \chi_{\max . f}\right)=\frac{\sin (\theta+\phi)}{2 \sin \theta \cos \phi}\left(1-\frac{y_{c, f}}{h}\right) \rho^{n_{\max } \cdot f}$.

These results depend on the angles only, and are independent of $h$.

The effective absorptance is

$$
\begin{aligned}
\alpha_{\mathrm{eff}}(\phi<\theta)=1- & \rho_{\mathrm{eff}}\left(\phi, \chi_{0} n\right)-\rho_{\mathrm{eff}}\left(\phi, \chi_{\max , n}\right) \\
& -\rho_{\mathrm{eff}}\left(\phi, \chi_{0, f}\right)-\rho_{\mathrm{eff}}\left(\phi, \chi_{\max . f}\right) .
\end{aligned}
$$

If $\phi=\theta$, eq (26) gives the same result as eq (20) for the $\phi>\theta$ case. This is expected because when $\phi=\theta$, no energy is actually directly incident on the near wall of the groove. If $\phi=0$, there can be no distinction between the two walls of the groove, and they will both have the same quantity of energy incident on them. In this case

$$
\rho_{\text {eff }}\left(\phi, \chi_{0, n}\right)=\rho_{\text {eff }}\left(\phi, \chi_{0, f}\right)
$$

and

$$
\rho_{\mathrm{eff}}\left(\phi, \chi_{\max , n}\right)=\rho_{\mathrm{eff}}\left(\phi, \chi_{\max . f}\right)
$$

\section{Comparison of the Theoretical and Experi- mental Results}

Measurements have been made to test the theoretical results presented above. The angular distribution of energy reflected in the plane of incidence by carefully prepared $V$-grooved surfaces, which were obtained from Bausch and Lomb, Inc., was studied as a function of angle of incidence. Details of the apparatus and the specimens may be found elsewhere [4].

Figure 2 shows the positions of the peaks in the reflected energy distribution as calculated with eqs

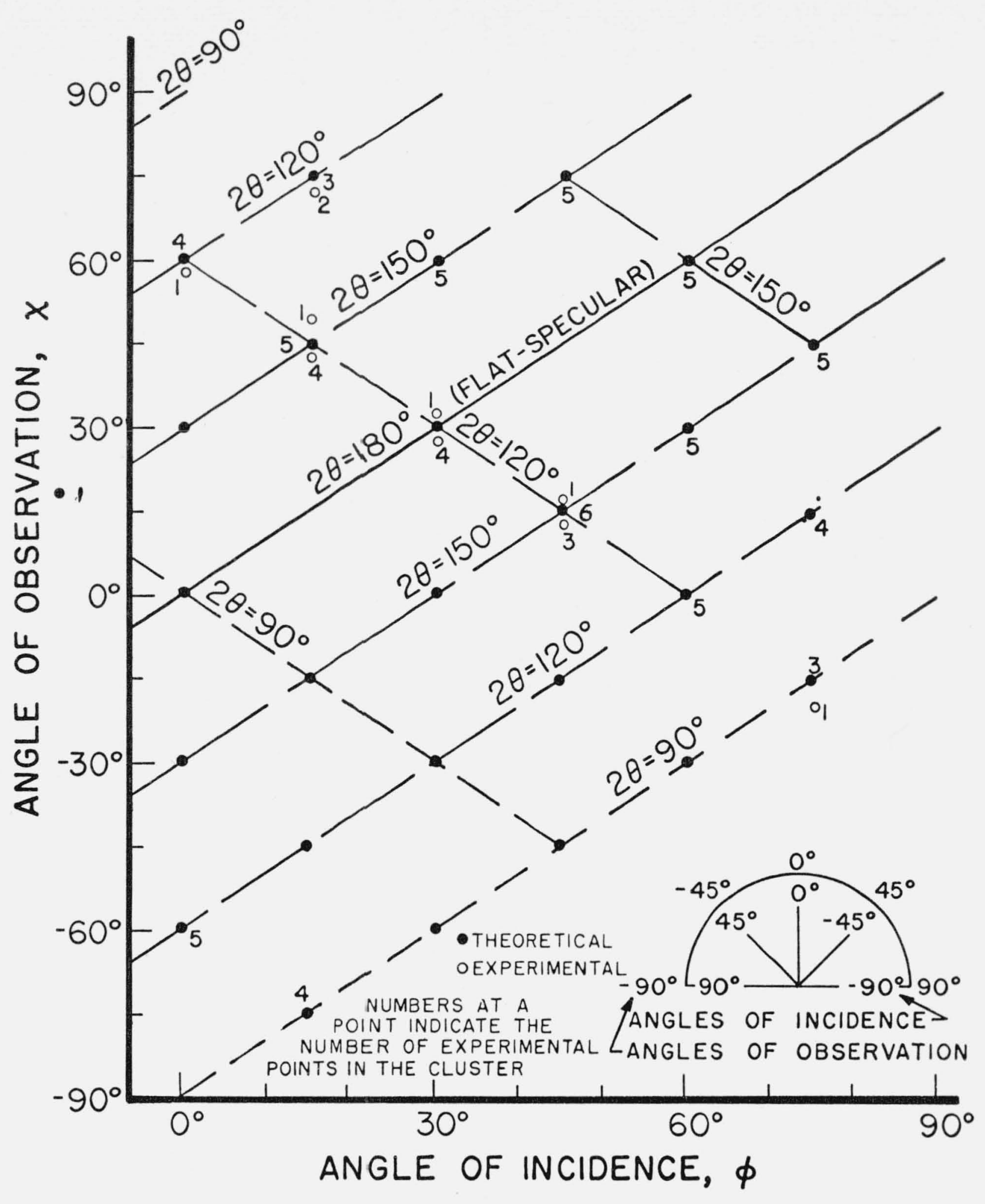

Figure 2. The positions of the peaks of energy reflected by the $\checkmark$-grooves with specular walls.

(4) and (7), for V-grooves having included angles of $90^{\circ}, 120^{\circ}$, and $150^{\circ}$. The experimentally determined positions are also shown in the figure, and they agree very well with the theoretical results.

In figures 3, 4, and 5, the theoretical and experimental values of the reflectance ratio, $R$, at the peaks of the reflected energy distribution are plotted as a function of angle of incidence, for the three values of groove angle. The reflectance ratio is defined as

$$
R=\frac{\rho_{\mathrm{eff}}\left(\phi_{i}, \chi_{i}\right)}{\rho_{\mathrm{eff}}\left(\phi_{j}, \chi_{j}\right)}
$$

In each figure, the point at which the experimental value of $R$ was made unity by choosing the measured value of $\rho_{\text {eff }}\left(\phi_{j}, \chi_{j}\right)$ as the reference is $;$.dicated. At these points the theoretical values of $\rho_{\text {eff }}\left(\phi_{j}, \chi_{j}\right)$ are unity.

Due to the nature of the apparatus, many of the reflected peaks could not be observed. The boxes in the figures are used to indicate which theoretical point the experimental points are to be compared with, wherever there is a possibility of confusion. The theoretical points at which the reflectance ratio vanishes at either grazing observation $\left(\chi=90^{\circ}\right)$ for nongrazing incidence, or at nongrazing observation for grazing incidence $\left(\phi=90^{\circ}\right)$ are the limiting cases obtained from eqs (4) and (7), but peaks cannot exist exactly at these angles, as is proven by Beckmann [1]. 


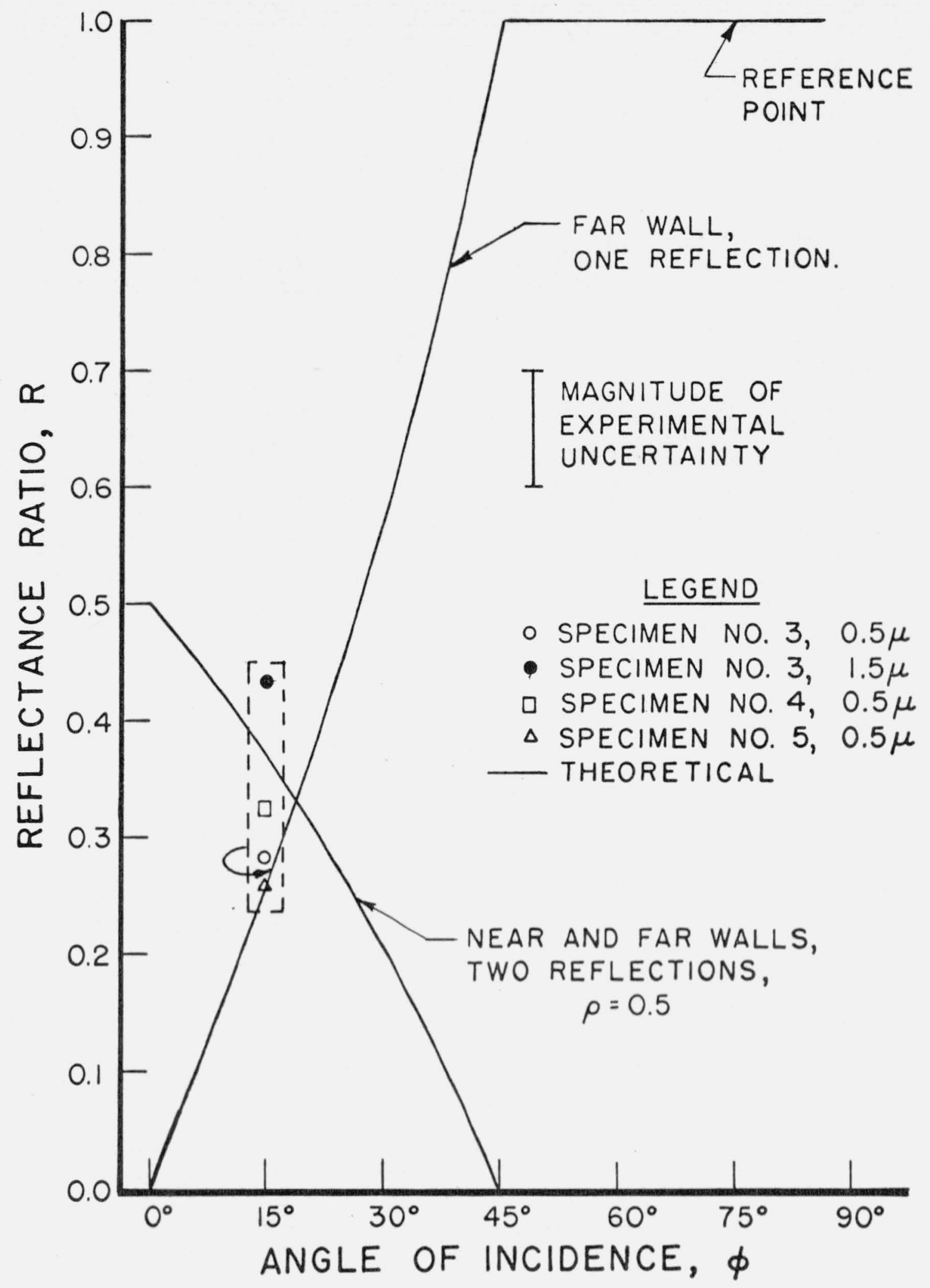

Figure 3. Comparison of theory and experiment for V-grooves with specular walls, $2 \theta=90^{\circ}$.

Except for a few cases of large deviations between the experimental and the theoretical values of $R$, figures 3,4 , and 5 show that reasonable agreement exists between them. The agreement is much better at $0.5 \mu$ than at $1.5 \mu$, since the assumption that the walls of the grooves reflect specularly becomes better as the wavelength is decreased. Theoretical curves for cases of more than one reflection were calculated using $\rho=0.5$.

\section{Discussion}

The assumption that the average reflectance of the groove wall surfaces is known is only rigorous when the wall reflectance is not a function of the angle of incidence on the wall. If the reflectance is a function of the angle of incidence on the wall, the calculations would have to be repeated, and terms such as $\rho^{n}$ would be changed to a product of $n$ different values of $\rho$, each one the reflectance of the groove wall at the angle of incidence of one of the $n$ reflections. Except for this the calculations are valid for monochromatic radiation of any wavelength, or for total radiation if the properties of the groove wall are independent of wavelength. ${ }^{3}$

${ }^{3}$ It should be noted that the only wavelength dependence in the analysis is due to the value of $\rho$ used.

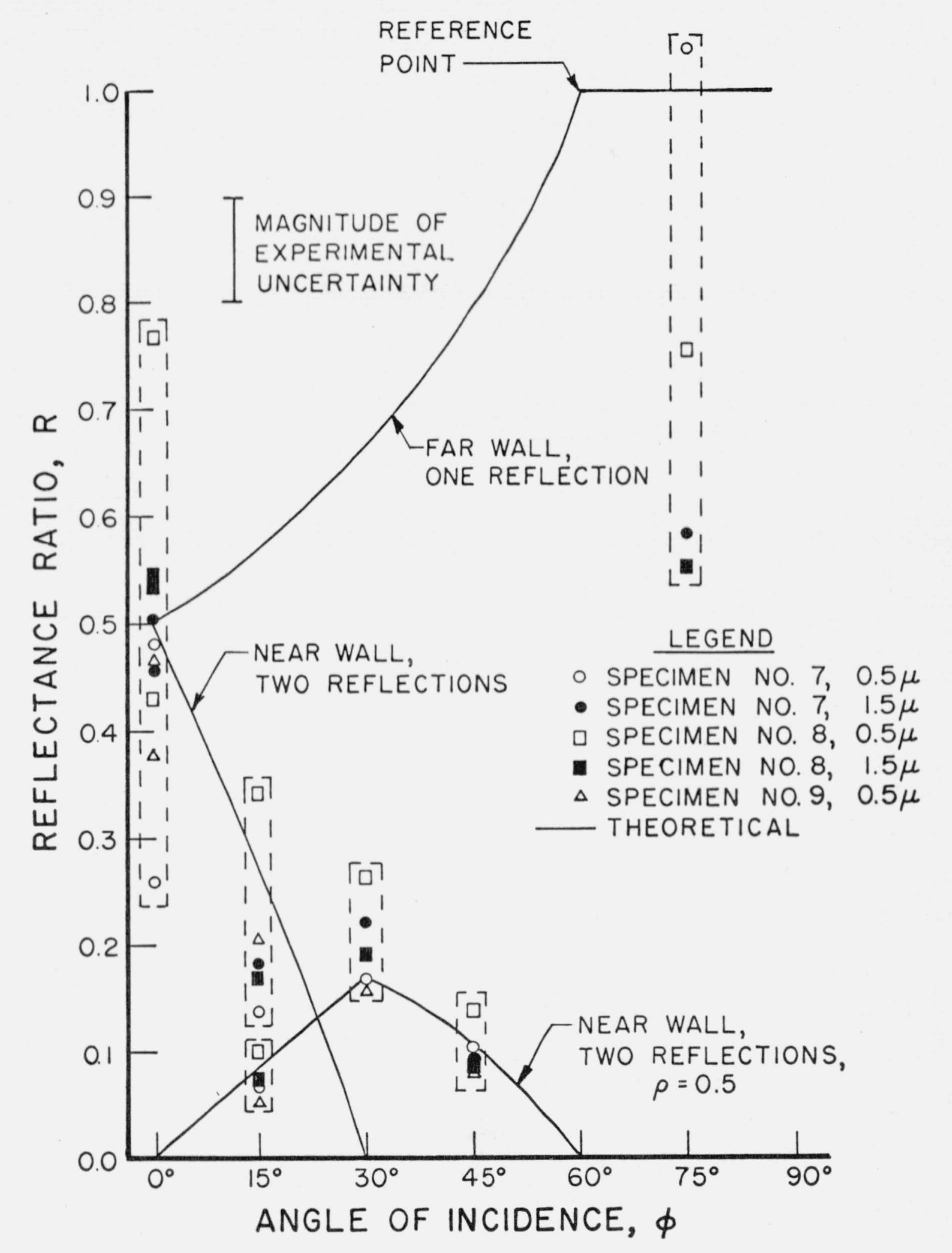

Figure 4. Comparison of theory and experiment for $\mathrm{V}$-grooves with specular walls, $2 \theta=120^{\circ}$.

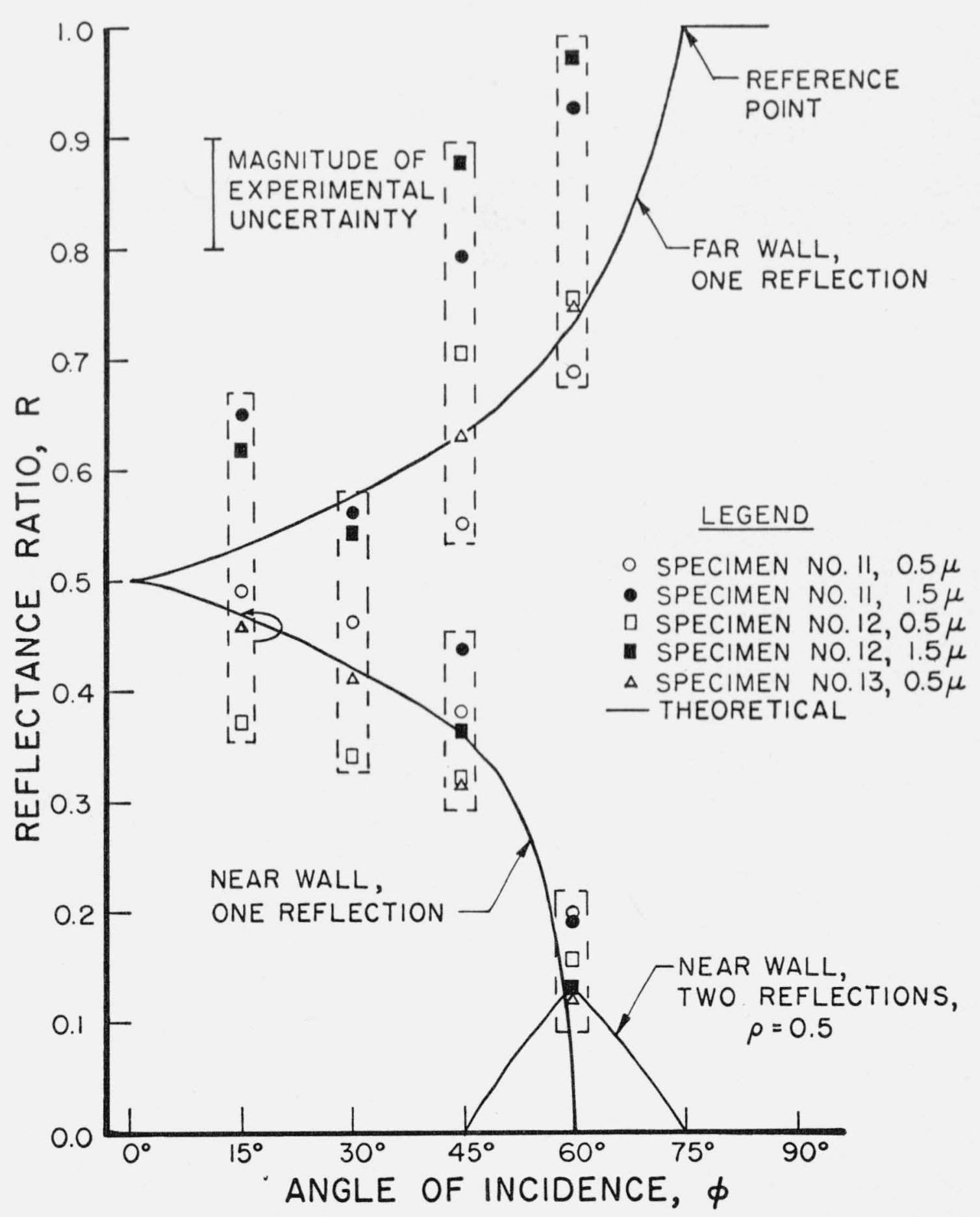

FIGURE 5. Comparison of theory and experiment for V-grooves with specular walls, $2 \theta=150^{\circ}$. 
If the groove walls have properties that are not independent of wavelength and calculations are desired for total radiation, another complication enters the situation. Let $\rho(\lambda)$ be the wall reflectance as a function of wavelength, and $I(\lambda)$ be the spectral distribution of the source, then any function $G(\rho(\lambda))$ can be calculated for total radiation by the use of the following expression:

$$
\{G(\rho(\lambda))\}_{\text {total }}=\frac{\int G(\rho(\lambda)) I(\lambda) d \lambda}{\int I(\lambda) d \lambda}
$$

where the integrations are performed over all wavelengths emitted by the source.

Any desired calculations can now be made for the apparent absorptance or reflectance when the walls are specular. It should be noted that sometimes an equation will yield absurd results which have no physical significance. Should this occur, the appropriateness of the parameters under consideration should be reexamined to ascertain their validity for the case under consideration.

A conclusion which may be drawn from the results is that the effective absorptance increases and the effective reflectances decrease as $\phi$ decreases. In the case $\phi>\theta$, there will always be a region at the vertex of the groove which will be in shadow, i.e., it will not be illuminated either directly or due to internal reflections. In the case $\phi<\theta$ the whole internal surface of the groove will be illuminated.

The author expresses his thanks to his major professor, Dr. Y. S. Touloukian for his advice and encouragement during the course of this work, and to the Purdue Research Foundation for support in the form of a David Ross Grant. Thanks are also due Dr. D. P. DeWitt for suggestions and stimulating discussions.

\section{Nomenclature}

$G=$ Any radiation function, as used in eq (30).

$h=$ The depth of a $\mathrm{V}$-groove.

$I=$ Energy per unit time carried by a beam of radiation. $n=$ The number of reflections within a specular V-groove.

$R=$ Reflectance ratio

$y=$ A distance measured vertically from the lip of a V-groove.

$\alpha=$ Absorptance.

$\theta=$ The half-angle of a $\mathrm{V}$-groove.

$\lambda=$ Wavelength.

$\rho=$ Reflectance.

$\phi=$ The angle of incidence.

$\chi=$ An angle of reflection.

$\psi=$ An angle defined in figure 1 .

$[]=$, Brackets indicating a quantity to be rounded off to the nearest higher integer.

\section{Subscripts}

$c=$ A critical value.

eff = An effective or apparent value of some quantity. $f, n=$ A quantity associated with the far or near wall of a $\mathrm{V}$-groove, respectively.

$i, j=$ Indexes.

$i, r=\mathrm{A}$ quantity associated with an incident or reflected beam, respectively.

$\max =\mathrm{A}$ quantity associated with the maximum value of $y$ on the far wall of a $V$-groove.

$0=$ A quantity associated with $y=0$.

\section{References}

[1] Beckmann, P., and Spizzichino, A., The Scattering of Electromagnetic Waves from Rough Surfaces, p. 33 (Pergamon and Macmillan, 1963)

[2] Sparrow, E. M., Eckert, E. R. G., and Jonsson, V. K., An enclosure theory for radiative exchange between specularly and diffusely reflecting surfaces, Trans. ASME, J. Heat Transfer 84C, No. 4, 294-300 (1962).

[3] Sparrow, E. M., and Lin, S. H., Absorption of thermal radiation in a V-Groove cavity. Int. J. Heat Mass Transfer 5, 1111-1115 (1962).

[4] Zipin, R. B., The Directional Spectral Reflectance of WellCharacterized Symmetric V-Grooved Surfaces, Ph. D. Thesis, Purdue University, 1965.

(Paper 70C4-238) 